\title{
Giardia duodenalis pathogenicity on immunosuppressed animal model
}

\author{
Wakid, M.H. ${ }^{1,2^{*}}$, Toulah, F.H. ${ }^{3}$, Mahjoub, H.A. ${ }^{4}$, Alsulami, M.N. ${ }^{3}$ and Hikal, W.M..$^{5,6}$ \\ ${ }^{1}$ Medical Laboratory Sciences Department, Faculty of Applied Medical Sciences, King Abdulaziz University, \\ Jeddah, Saudi Arabia \\ ${ }^{2}$ Special Infectious Agents Unit, King Fahd Medical Research Center, Jeddah, Saudi Arabia \\ ${ }^{3}$ Biology Department, College of Science, University of Jeddah, Jeddah, Saudi Arabia \\ ${ }^{4}$ Biology Department, College of Sciences and Arts, King Abdulaziz University, Rabigh, Saudi Arabia \\ ${ }^{5}$ Biology Department, Faculty of Science, University of Tabuk, Tabuk, Saudi Arabia \\ ${ }^{6}$ National Research Centre, Water Pollution Research Department, Giza, Egypt \\ *Corresponding author e-mail: mwakid@kau.edu.sa. \\ Received 9 February 2020; received in revised form 2 July 2020; accepted 3 July 2020
}

\begin{abstract}
Giardiasis is the major water-borne diarrheal disease present worldwide caused by the common intestinal parasite, Giardia duodenalis. This work aims to investigate the effect of $G$. duodenalis infection pathogenicity in immunosuppressed animals through histopathological examination. A total of $45 \mathrm{BALB} / \mathrm{c}$ mice were divided into four groups; G1 (negative control), G2 (healthy animals exposed to Giardia); G3 (immunosuppressed animals exposed to Giardia), and G4 (non-exposed immunosuppressed animals). Our study revealed that G3 was the most affected group with an infection rate of $100 \%$. The animals showed general weakness, soft stool, and high death rate with severe histopathological changes in the duodenum and mild degenerative changes in hepatic tissues. In G2, the maximal lesions in both duodenum and liver were on the $11^{\text {th }}$ day. We spotted damage in the villi, edema in the central core, and submucosa, in addition to increased cellular infiltration with inflammation in lamina propria. The presence of the parasites within the villi and the lumen was clear. Most of the hepatocytes revealed hydropic and fatty changes, also dilated congested central veins and edema were observed. G3 changes were more intense than G2 with massive Giardia trophozoites between the intestinal villi, lumen, and extensive fatty liver degeneration. Immune suppression plays a significant role in the severity of injury with the Giardia parasites in duodenum and liver cells.
\end{abstract}

\section{INTRODUCTION}

The flagellate protozoan parasite Giardia duodenalis (known as G. lamblia and $G$. intestinalis) is among the common parasites infecting the small intestine of humans and various animals. The reported infection prevalence ranges from $2-5 \%$ to $20-30 \%$ in industrial and developing countries, respectively. Giardiasis remains among the major water-borne diarrhea-causing diseases all over the world, and each year infects about 280 million people with symptomatic 500,000 , mainly in poor regions, and most outbreaks are attributable to drinking untreated water (WHO, 2020; Torgerson et al., 2015).

The excreted cysts remain infectious for several months in cold water and humid conditions. Infection can lead to asymptomatic intestinal colonization, acute or chronic diarrheal disease. These disparities are likely associated with several factors, including the patient's age, immunological and nutritional status, history of previous exposures, in addition to the severity of parasite strains (Shalaby \& Wakid, 2014). Fatty diarrhea, abdominal pain, distension, vomiting, and nausea in children are more acute, while chronic infection may 
end with weight loss accompanied by malabsorption. Giardia parasite can penetrate the lamina propria up to the liver, causing hepatitis and cholangitis. Rarely, the parasites infect the stomach in the case of achlorhydria leading to extra-intestinal manifestations, allergic symptoms, and reactive arthritis (Cantey et al., 2011). Some reported cases of giardiasis were among homosexual men, immunocompetent and immunocompromised patients. In addition, giardiasis in children living in developing countries can cause growth retardation (de la Guardia et al., 2011). The main aim of this study is to evaluate the pathogenicity of $G$. duodenalis infection through the histopathological examination of the experimentally immunosuppressed animal model.

\section{MATERIALS AND METHODS}

\section{Experimental animals}

We conducted this study on 45 male mice of BALB/c strain (between 3-4 weeks old, weighing $20 \pm 2 \mathrm{gm}$ ) that housed in pathogenfree conditions at the animal house of King Fahd Medical Research Center (KFMRC).

\section{Cysts of G. duodenalis}

Cysts were obtained from a fresh stool sample of heavily infected patient attending King Abdulaziz University Hospital and confirmed by modified formal ether technique and trichrome staining (Wakid et al., 2009).

\section{Infection of animals with Cysts}

Cysts were washed several times with sterile normal saline before intraesophageal inoculation of each animal with $10^{5}$ cysts in $200 \mu \mathrm{L}$ (Ismail et al., 2017).

Immunosuppression of animals by Dexamethasone

The immunosuppressive therapy, dexamethasone sodium phosphate (Miller \& Schaefer, 2007) was purchased in the form of liquid ampoules (8 mg/2 ml, EIPICO, Egypt). Animals were injected intramuscularly $(0.5$ $\mathrm{mg} / \mathrm{kg} /$ day) daily beginning two weeks before infection and continuing through the end of the study (Tu \& Lai, 2006).

\section{Experimental design}

Animals were divided into the following four groups; (G1) negative control mice $(\mathrm{n}=5)$; (G2) exposed normal mice; $(n=15)$; (G3) exposed immunosuppressed mice $(\mathrm{n}=15)$; and (G4) non- exposed immunosuppressed control group $(n=10)$. All animals were observed in terms of vitality, activity, appetite, skin condition, as well as the occurrence of soft stool, abdominal distension, and deaths. Subsequently, fresh fecal pellets were collected every 24 hours from post-infection (PI) day 2 and examined for the presence of G. duodenalis parasites till day 21 .

\section{Parasitological assay}

3-5 fecal pellets were collected individually from each animal every other day until the 21st day. About $0.1 \mathrm{gm}$ of fecal sample was mixed thoroughly with $1 \mathrm{~mL}$ formal saline, then stained with iodine and cysts counted using a hemocytometer (Shukla et al., 2012). Rate of animals acquired the infection, the number of days required to shed the parasite cysts after infection (prepatent period), and the time for the complete disappearance of the parasites (patent period) was recorded for each applicable sample.

\section{Histopathological examination}

Each animal was sacrificed by ether inhalation, then right lobe of the liver, the upper $6 \mathrm{~cm}$ of the duodenum and proximal jejunum were quickly removed and fixed in $10 \%$ formal saline. All fixed specimens were dehydrated, then processed for embedding in paraffin wax, and finally sectioned (3-5 $\mu \mathrm{m}$ thicknesses) using a rotary microtome. For detection of the histopathological changes, each histological section was stained with hematoxylin and eosin (H\&E), then examined microscopically. 
Table 1. Infection rate, prepatent period and death rate in the studied groups

\begin{tabular}{lccc}
\hline Group & $\begin{array}{c}\text { Infection Rate } \\
\text { No. (\%) }\end{array}$ & $\begin{array}{c}\text { Prepatent Period } \\
\text { (days) }\end{array}$ & $\begin{array}{c}\text { Death Rate } \\
\text { No. }(\%)\end{array}$ \\
\hline G1 $(\mathrm{N}=5)$ & NA & NA & $0(0)$ \\
G2 $(\mathrm{N}=15)$ & $13(86.6)$ & $3-5$ & $2(13.3)$ \\
G3 $(\mathrm{N}=15)$ & $15(100)$ & $1-3$ & $15(100)$ \\
G4 $(\mathrm{N}=10)$ & NA & NA & $3(30)$ \\
$P$ value & $>0.05$ & $<0.05$ & $<0.05$ \\
\hline
\end{tabular}

NA; not applicable.

\section{Statistical analysis}

All data were statistically analyzed using SPSS V20 software for windows. Differences among groups were tested for significance by ANOVA. Continuous data were reported by count and standard deviation (SD). Chisquared test was used for the comparison of significance between groups, and $\mathrm{P}$ value $(<0.05)$ considered statistically significant.

\section{Ethical approval}

All procedures performed on animals were in accordance of ethical standards and guidelines of KFMRC review board, King Abdulaziz University, at which the study was approved and conducted.

\section{RESULTS}

\section{General observations}

The variation observed in the manifestations of infection in the different groups of mice concerning clinical symptoms such as loss of appetite, poor performance, general weakness, hair loss, and the occurrence of soft stool. All mice in the control group (G1) were subjected to similar general observations and histological examination. Since PI day 1 , there were notable differences in symptoms that appeared in groups G2 and G3. After PI day 7, the status of mice deteriorated sharply since all suffered from extreme weakness in addition to a hair loss around the eyes. In G2, animals suffered from the occurrence of soft stool after PI day 7 . We observed that G4 mice suffered from weakness and lethargy during the period of the experiment.
The infection rate among the immunosuppressed group (G3) was higher than the healthy group exposed to Giardia (G2) (100\%, $86.6 \%)$, respectively, without significance ( $\mathrm{P}>0.05)$ (Table 1). The prepatent periods were 1-3 and 3-5 days in G3 and G2, respectively. Furthermore, death rates were $0,13,100$ and $30 \%$ among G1, G2, G3 and G4, respectively.

\section{Mean number of excreted $G$. duodenalis cysts}

As shown in Table 2, the average numbers of $G$. duodenalis parasites at PI day 3 in G2 were ten times less than G3. The parasites continued in the multiplication and reached the highest rate at PI day 11, after that, we noted major decrease and finally disappeared completely in all mice at PI day 19. On the other hand, we were able to detect $G$. duodenalis parasites at PI day 1 in G3. The numbers increased to the highest rate at

Table 2. Mean number of cysts \pm SD/HPF

\begin{tabular}{ccc}
\hline PI day & G2 & G3 \\
\hline 1 & 0 & $33.6 \pm 6.02$ \\
3 & $4 \pm 1$ & $43.2 \pm 8.40$ \\
5 & $14.6 \pm 1.14$ & $77.8 \pm 4.20$ \\
7 & $35.6 \pm 3.97$ & $104.4 \pm 9.20$ \\
9 & $49.2 \pm 7.72$ & $90 \pm 6.67$ \\
11 & $78.2 \pm 4.91$ & $87.4 \pm 6.94$ \\
13 & $35.2 \pm 5.31$ & $82.6 \pm 7.12$ \\
15 & $14.2 \pm 9.17$ & $72.6 \pm 5.50$ \\
17 & $4.8 \pm 3.49$ & $72.8 \pm 5.63$ \\
19 & 0 & $* *$ \\
21 & 0 & $* *$ \\
\hline
\end{tabular}

** Death of animals. 
PI day 7 , then declined gradually till PI day 17 , and all animals died the next days.

\section{Histopathological study on the duodenum}

Figure 1 shows the normal appearance of duodenum in negative control group (G1) without any villus atrophy or fusion, and no inflammatory response in the lamina propria. Histopathological changes occurred in G2 since PI day 4 with some of the short villi and infiltration with inflammatory cells, in addition to edema in the connective tissue of villi central core (Fig. 2A). At the PI day 11, most villi decreased in height with edema in the submucosa, lymphoid infiltration, increase in goblet cells, congestion, and extravasation of blood with the occurrence of Giardia parasitization between villi (trophozoites in crescent, oval or teardrop shapes) (Fig. 3A). Looking at G3 duodenal sections, we found significant histopathological changes since PI day 4, where most of the villi ruptured and decreased in height, hyperplasia in some areas with the appearance of Giardia trophozoites between villi (Fig. 2B). The damage of microvillus intensified at PI day 11 , with dilation of blood vessels, swelling edematous submucosal area (intestinal angioedema), and marked increase of goblet cells with necrosis of the nuclei (Fig. 3B). In all G4 samples, the changes occurred during the different periods (PI days 4 and 11) with hemorrhage, lymphoid infiltration, and an increase in the thickness of the muscle layer (Fig. 3C).

\section{Histopathological study on the liver}

The histopathological finding of the negative control group (G1) showed normal polygonal lobules with hepatocytes arranged in cords radiating outward from the central vein (Fig. 4A). At PI day 4, G2 cells seem like healthy cells, while infiltration of inflam-

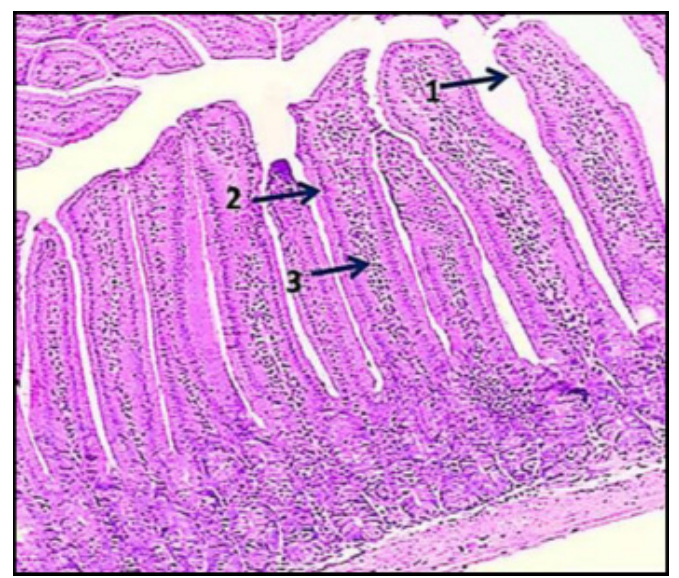

Figure 1. Cross-section in the duodenum for G1 illustrates the form of high and long villi (1) the integrity of the epithelial cells lining the villi (2) surrounding a core of lamina propria (3). (H\&E stain, 100X).
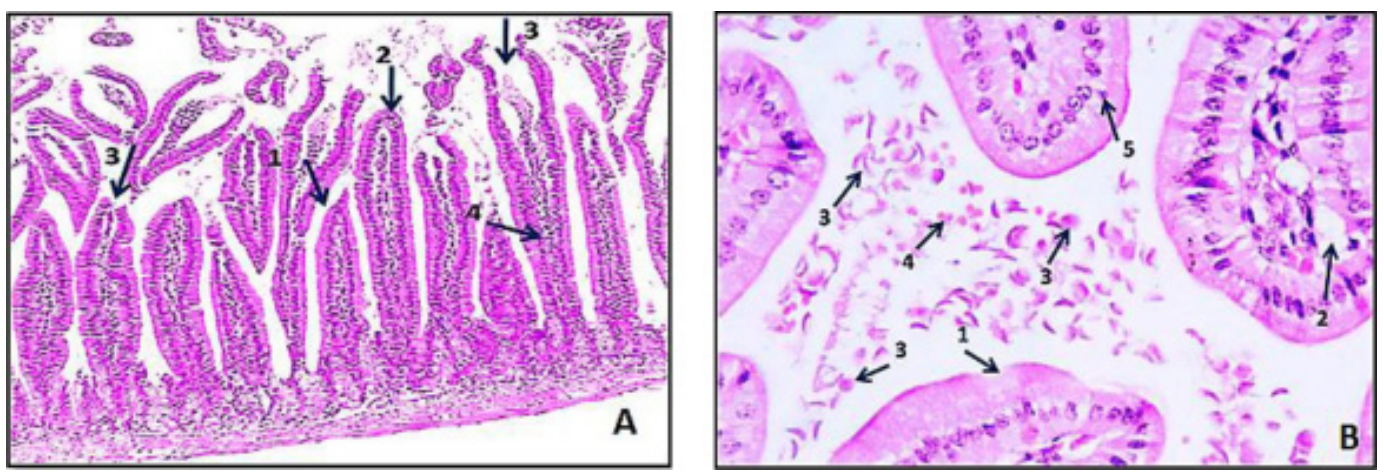

Figure 2. The cross-section in the duodenum at PI day 4, for G2 (A) and G3 (B). G2 revealed a shortage of some villi (1), normal form (2), erosion of epithelial cells lining the top of some villi (3), and an increase of inflammatory cells infiltrate in the lamina propria core of the villi (4). G3 shows erosion in the edge of cells lining the villi (1), mucus droplets in the connective tissue of the core of the villi (2), the spread of the parasite strongly between villi, with different shapes as a crescent and others oval or teardrop (3), the existence of inflammatory cells infiltrations (4). Note the presence of parasites inside the surface mucous of the epithelial cells (5). (H\&E stain, A; 100X, B; 400X). 

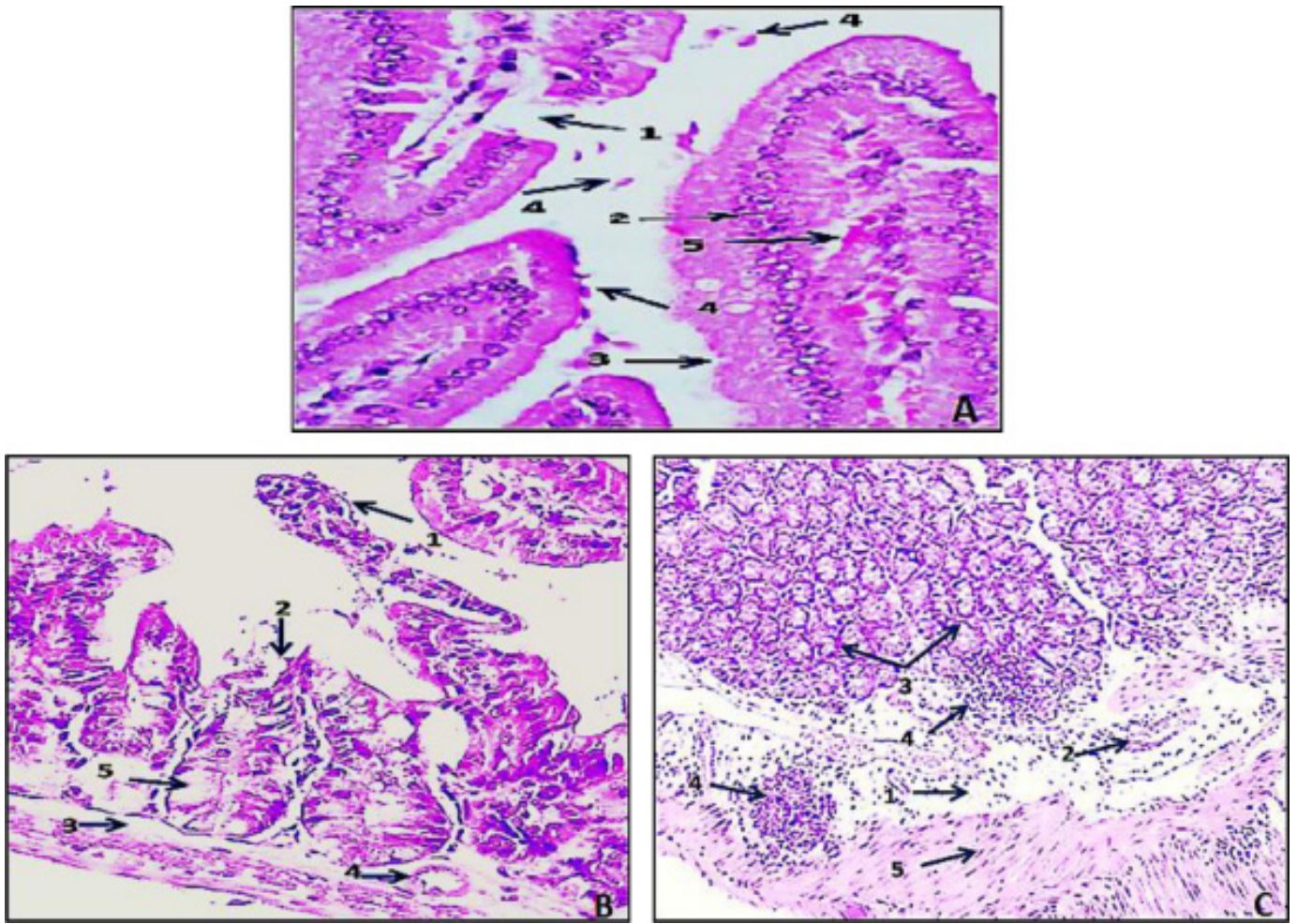

Figure 3. The cross-section in the duodenum at PI day 11, for; G2 (A) shows erosion of the epithelial cells lining the villi (1), areas of hyperplasia (2), atrophy in the edges (3), parasite's different shapes on the villi mucosa (4), congestion and extravasation of blood in the lamina propria core of the villus (5); G3 (B), disorganized villi and loss of the normal structure of villi (1), while some villi have dislodged tips (2), swelling edematous submucosal area (3), dilated blood vessels (4) and marked increase in the goblet cells with necrosis of nuclei (5); G4 (C), swelling edematous submucosal area (1), dilated blood vessels (2), an apparent increase in crypt (3), nodular lymphocytic infiltration (4). Note the thickness in the muscle layers (5). (H\&E stain, A; 400X, B; 200X, C; 100X).
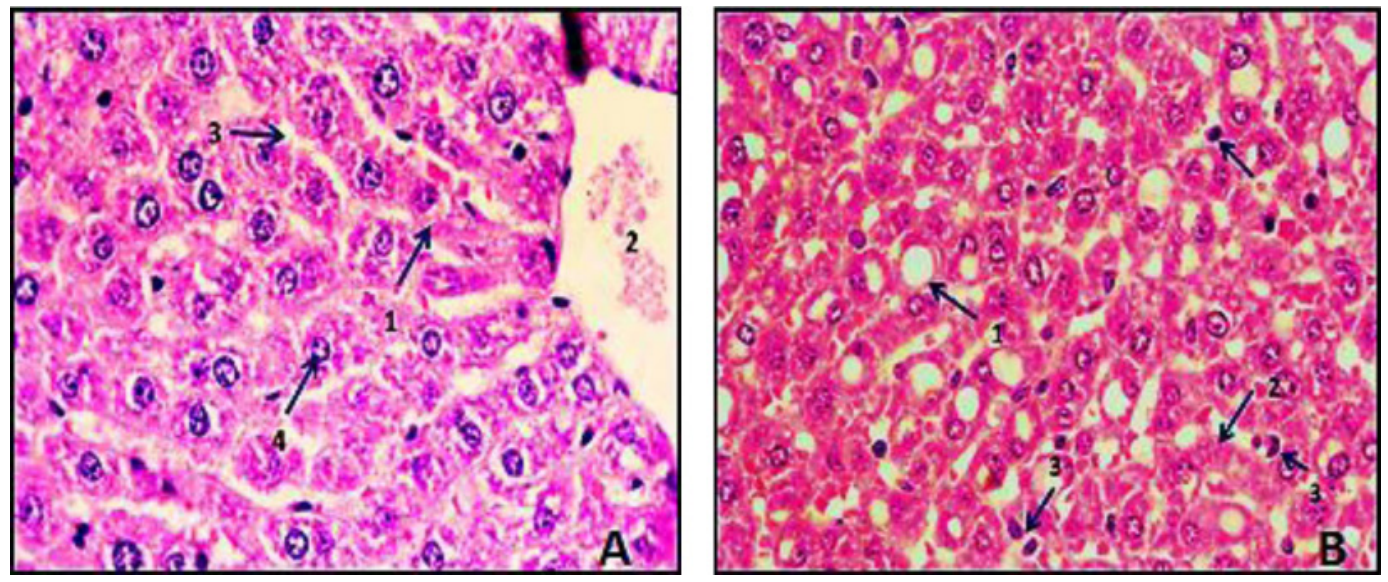

Figure 4. Cross-section in the liver (A) from G1 illustrates the regularity of the cells in the form of normal polygonal classic hepatic lobules with tightly packed cords of hepatocytes (1), radiating from central veins (2), separated by blood sinusoids (3), hepatocytes with vesicular central nuclei and acidophilic cytoplasm; (B) at PI day 4 for G3 revealed large hepatocytes with lightly stained foamy vacuolated cytoplasm (1), some necrosis (2) and parasites between liver cells (3). (H\&E stain, 100X). 

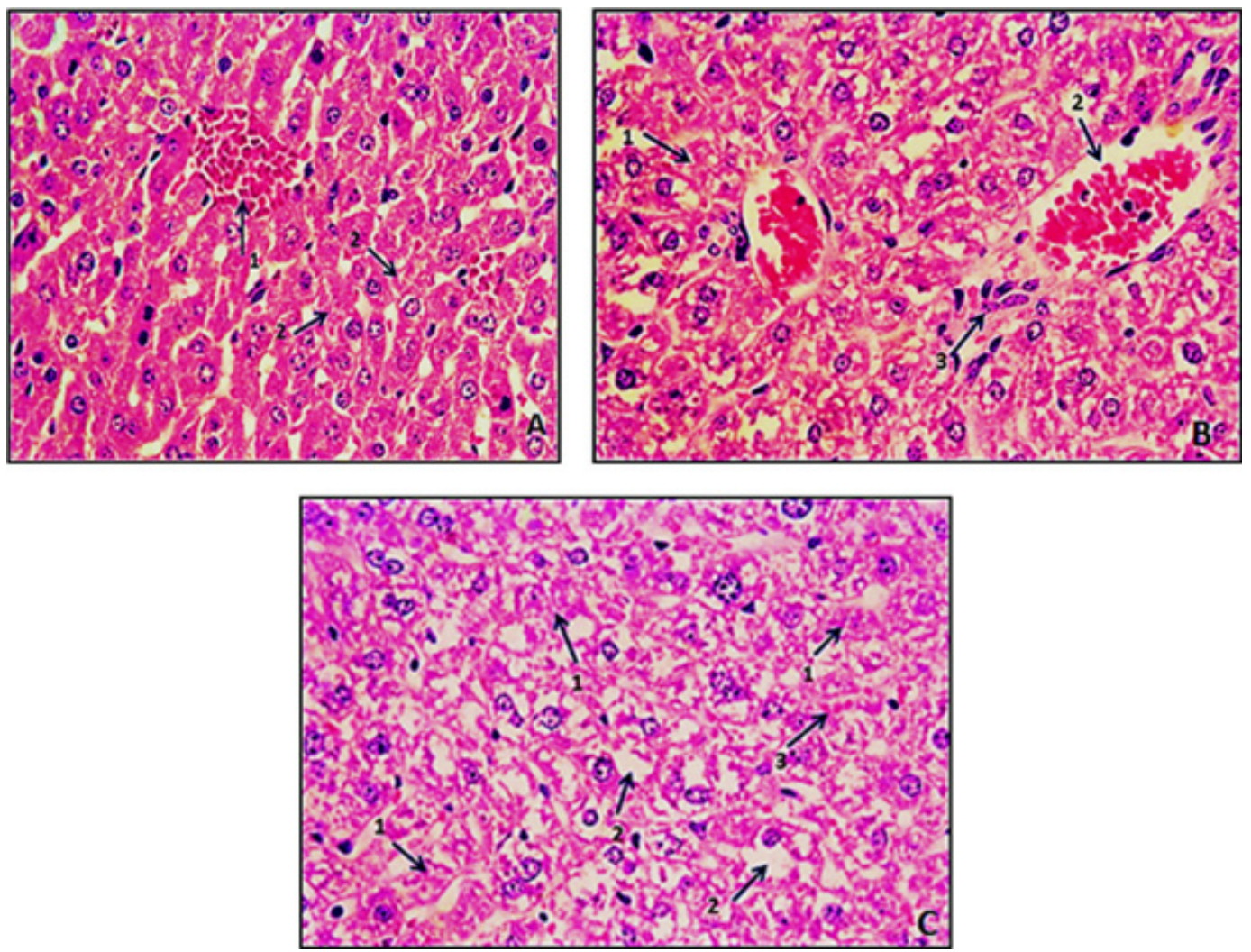

Figure 5. Cross-section in the Liver at PI day 11 for G2 (A), G3 (B) and G4 (C). G2 shows dilated congested central vein (1) necrosis in some cells (2). G3, irregularly arranged hepatocytes with loss of the normal architecture of hepatic lobule with the occurrence of necrosis in most cells (1) dilated congested central vein (2) leaching of blood cells with the spread of the parasite (3). G4, massive distortion of the normal liver architecture with the occurrence of necrosis in most cells (1) Liver sinusoids are markedly dilated (2) and congested (3). (H\&E stain, 100X).

matory cells, tissue necrosis with hydropic degeneration during PI day 11 (Fig. 5A).

In G3, there was evidence for expanded hepatic portal veins, infiltration with blood cells, and foamy vacuolated cytoplasm with the appearance of the parasites between the liver cells on PI day 4 (Fig. 4B). On PI day 11 (Fig. 5B), cell lines diverged from each other with the emergence of ascites between cells. The changes in the cells of the liver in the eleventh day where there is a marked loss of the normal liver architecture with variable hepatocellular changes. Some hepatocytes are non-nucleated and dilated congested central vein with the leaching of blood cells and the spread of the parasite. We observed massive distortion of the normal liver architecture with necrosis in G4. Liver sinusoids were markedly dilated and congested (Fig. 5C).

\section{DISCUSSION}

Male BALB/c mice were used in this study as the experimental and clinical data indicated that males' cellular and humoral immune responses are more potent with higher immunoglobulins (Hamam et al., 2017). In our study, mice in G1 were asymptomatic, while mice in G2 suffered from soft stool. On the other hand, infected immunosuppressed mice in G3 suffered from soft stool with a bulge in the abdominal area, as well as lethargy, sleep, and lack of movement with hair loss, especially around 
eyes, while G4 suffered lethargy and weakness. The symptoms of infection are distinct because of the ability of the immune system in G2 to reduce symptoms of the disease relative to immunosuppressive mice in G3. Prior evidence suggests that the clinical manifestations of giardiasis depend on the immunological status of the host (Ismail et al., 2017). Therefore, immunosuppressed hosts have more severe clinical signs than normal healthy hosts.

The current study showed that the number of cysts in fecal samples from G2 peaked at PI day $11(\mathrm{n}=78.2)$, and no parasites were detected by PI days 19 and 21 . This pattern of infection is consistent with those previously reported (Mahmoud et al., 2014; Ismail et al., 2017). Contrary to our finding, Dreesen et al. (2014) described the infection pattern with an early peak around PI day 6 , and the infection ended three weeks PI. Our results may indicate that the deficiency in immunity has a vital role in the infection intensity, as the number of cysts in stool from infected immunosuppressed animals (G3) was detected early with a peak at PI day $7(n=104)$ compared to G2 $(n=36)$. Prior research involving diabetic mice has suggested that diabetes might suppress immune response leading to an intensified parasite burden in the small intestine regardless of the infection phase (Toulah et al., 2016). Innate immunity mechanisms are the first line of defense against the parasite, and the release of cytokines and the production of specific antibodies against parasite antigens activate the adaptive immune response (LopezRomero et al., 2015).

The prepatent period in G2 was from 3-5 days, and in G3, the period was from 1-3 days, and this result agrees with previous studies (EL-Shewy et al., 2005). None of the G2 animals died during our research; moreover, all mice in G3 died on PI day 19, indicating the role of immunosuppression, as we suggested above.

Our findings showed clear histological changes in the intestines of infected mice with Giardia at PI day 11 with a decrease in the length of the villi. Furthermore, an apparent increase of goblet cells, damage in the epithelial cells with the loss of nuclei, and increase infiltration of inflammatory cells with the presence of the parasite in the cavity of the intestines and between villi were observed. As has been suggested previously, the microvillus alterations are attributed to the adhesion of sucking discs of trophozoites with intestinal epithelium, infection stage, secretion of enterotoxins and cysteine protease or the surface mannose-binding lectin (Mahmoud et al., 2014; Ma'ayeh et al., 2017; Liu et al., 2018; Allain et al., 2019). On the other hand, another study did not find any changes in the villi or the activity of the intestines (Hewan-Lowe et al., 1997).

With necrosis of all villi cells, the duodenal tissue changes increased in G3 as the parasite density increased and continued throughout the study. In contrast to our observation, Ismail et al. (2017) reported that there were no significant histopathological changes in the small intestinal tissues in both immunocompetent and immunosuppressed infected albino mice except some inflammatory cellular infiltration and increase in goblet cell number. Similarly, another study explained that little to no mucosal inflammation was detected during giardiasis, as trophozoites do not invade the epithelial layer (Cotton et al., 2015). We were able to observe shortening in the villus height in all infected groups with more effect in the immunosuppressed animals (G3). A study conducted by Ventura et al. (2013) reported similar findings. The decrease in number and size of the villi have been linked to the decrease in production of enterocytes in the villi (Cotton et al., 2011). These results may suggest that the shortening of villi in severe intestinal giardiasis is the precursor for decreasing the mucosal absorption ability as a result of immunosuppression. In G4, we obtained similar results to those of EL-Shewy \& Eid (2005), which reported that there is a shortage of villi with submucosal edema, cellular infiltrates in the connective tissues of villi lamina propria ranging from simple to severe.

In the current study, we observed a slight increase in goblet cells of G2, while a marked improvement was found in the intestinal villi of G3. This notable increase in goblet cells 
known to be part of the protection mechanism against mucosal pathogens.

As the next step for further understanding the pathogenicity of giardiasis, we assessed the effect on liver cells of the studied animal groups. Liver cells in G2 at PI day 4 were like healthy cells except for the infiltration of inflammatory cells, and tissue necrosis with hydropic degeneration during PI day 11. These observations agree with Astiazarán García et al. (2010), with the occurrence of granulomatous hepatitis and cholangitis. Other researchers reported hydropic or eosinophilic degeneration and chronic venous congestion (Awadalla et al., 1995; Toulah et al., 2016). Changes that occurred in G2 may suggest the role of toxins and antigens of the parasite absorbed by the villi of the intestine and then reach the liver via portal venous tract (Samra et al., 1988).

When we further assessed the effect of infection on G3 at PI day 4, we observed expanded hepatic portal veins, infiltration with blood cells, and fatty degeneration with clear presence of the parasite between liver cells. Next, at PI day 11, we noticed cell lines diverged from each other with the emergence of edema between cells. These changes might be explained similarly as G2, in addition to the reducion factor of dexamethasone on the antiparasitic effect of the immune system. Interestingly, at PI day 11 , the parasite among liver cells exhibited dark color and atypical shapes in comparison with the intestinal infection. This can be potentially explained by the fact that eccentric forms of the parasite reach the liver through the biliary tract and can be detected in bile secretion (Aronson $e t$ al., 2001). Other workers suggested that the existence of parasite in the liver through penetrating the lamina propria of the small intestine or the surrounding tissue then move to the portal venous tract (Hewan-Lowe $e t$ al., 1997; Buret, 2007). On the other hand, the mutations formality on the active parasite such as loss of flagella and sucking disk were attributed to "the lack of need" in the new environment in the liver. Thus, difficulty in identification and the dark color of the parasite are attributed to coarse-grained materials of carbohydrates stored in the cytoplasm of the parasite as a result of adaption to this new environment (Salem et al., 1989).

\section{CONCLUSION}

We report herein that immune deficiency plays an essential effect in the injury with intestinal parasites that may be associated with the occurrence of severe infection. The ability of Giardia parasites to penetrate the mucous membrane of the small intestine and reach the liver is not necessarily to the appearance of symptoms as a guide to the incidence, with more susceptibility to Giardia infection in the immunosuppressed host. Dexamethasone revealed side effects on the injured tissues of infected immunosuppressed mice.

\section{Conflict of interest}

The authors declare that there is no conflict of interest.

Acknowledgements. The present study was financially supported by a research grant from King Abdulaziz City for Science and Technology, Saudi Arabia. King Fahd Medical Research Centre, especially the Animal House Unit for their beneficial help and cooperation.

\section{REFERENCES}

Allain, T., Fekete, E. \& Buret, A.G. (2019). Giardia cysteine Proteases: the teeth behind the smile. Trends in Parasitology 35: $636-648$.

Aronson, N.E., Cheney, C., Rholl, V., Burris, D. \& Hadro, N. (2001). Biliary giardiasis in a patient with human immunodeficiency virus. Journal of Clinical Gastroenterology 33: 167-170.

Astiazarán-García, H., Lopez-Teros, V., Valencia, M.E., Vazquez-Ortiz, F., SoteloCruz, N. \& Quihui-Cota, L. (2010). Giardia lamblia infection and its implications for vitamin A liver stores in school children. Annals of Nutrition and Metabolism 57: 228-233. 
Awadalla, H., El-Gowhary, S., Sadaka, H. \& Khalifa, A. (1995). Aminosidine sulphate in experimental giardiasis. Journal of the Egyptian Society of Parasitology 25: 53-61.

Buret, A.G. (2007). Mechanisms of epithelial dysfunction in giardiasis. Gut 56: 316-317.

Cantey, P.T., Roy, S., Lee, B., Cronquist, A., Smith, K., Liang, J. \& Beach, M.J. (2011). Study of nonoutbreak giardiasis: novel findings and implications for research. The American Journal of Medicine 124: 1175.

Cotton, J.A., Amat, C.B. \& Buret, A.G. (2015). Disruptions of host immunity and inflammation by Giardia duodenalis: potential consequences for co-infections in the gastro-intestinal tract. Pathogens 4: 764-792.

Cotton, J.A., Beatty, J.K. \& Buret, A.G. (2011). Host parasite interactions and pathophysiology in Giardia infections. International Journal Parasitology 41: 925933.

de la Guardia, R.D., Lopez, M.B., Burgos, M. \& Osuna, A. (2011). Purification and characterization of a protein capable of binding to fatty acids and bile salts in Giardia lamblia. Journal of Parasitology 97: 642-647.

Dreesen, L., De Bosscher, K., Grit, G., Staels, B., Lubberts, E., Bauge, E. \& Geldhof, P. (2014). Giardia muris infection in mice is associated with a protective interleukin 17A response and induction of peroxisome proliferator-activated receptor alpha. Infection and Immunity 82: 3333-3340.

EL-Shewy, K.A. \& EID, R.A. (2005). In vivo killing of Giardia trophozoites harbouring bacterial endosymbionts by intestinal Paneth cells: an ultrastructural study. Parasitology 130: 269-274.

Hamam, G.G., Fekry, H. \& Gawad, S.A. (2017). Effect of pirfenidone on cardiac complications in a model of Kawasaki disease in female Balb/C mice: histological and immunohistochemical study. Egyptian Journal of Histology 40: 290-302.
Hewan-Lowe, K., Furlong, B., Sims, M. \& Schwartz, D.A. (1997). Coinfection with Giardia lamblia and Enterocytozoon bieneusi in a patient with acquired immunodeficiency syndrome and chronic diarrhea. Archives of Pathology \& Laboratory Medicine 121: 417-422.

Ismail, H.I., Ashour, D.S., Saad, A.E. \& Rizk, O.K. (2017). Impact of immune suppression on histopathological and immunological parameters of mice experimentally infected with Giardia lamblia. Jourmal of the Egyptian Society of Parasitology 47: 197-206.

Tu, W.C. \& Lai, S.C. (2006). Angiostrongylus cantonensis: efficacy of albendazoledexamethasone co-therapy against infection-induced plasminogen activators and eosinophilic meningitis. Experimental Parasitolology 113: 8-15.

Liu, J., Ma'ayeh, S., Peirasmaki, D., LundströmStadelmann, B., Hellman, L. \& Svärd, S.G. (2018). Secreted Giardia intestinalis cysteine proteases disrupt intestinal epithelial cell junctional complexes and degrade chemokines. Virulence 9: 879894.

Lopez-Romero, G., Quintero, J., AstiazaránGarcía, H. \& Velazquez, C. (2015). Host defences against Giardia lamblia. Parasite Immunology 37: 394-406.

Ma'ayeh, S.Y., Liu, J., Peirasmaki, D., Hörnaeus, K., Bergström, Lind, S., Grabherr, M., Bergquist, J. \& Svärd, S.G. (2017). Characterization of the Giardia intestinalis secretome during interaction with human intestinal epithelial cells: The impact on host cells. PLoS Neglected Tropical Diseases 11: e0006120.

Mahmoud, A., Attia, R., Said, S. \& Ibraheim, Z. (2014). Ginger and cinnamon: can this household remedy treat giardiasis? Parasitological and histopathological studies. Iranian Journal of Parasitology 9: $530-540$. 
Miller, T.A. \& Schaefer, F.W. 3rd. (2007). Changes in mouse circulating leukocyte numbers in $\mathrm{C} 57 \mathrm{BL} / 6$ mice immunosuppressed with dexamethasone for Cryptosporidium parvum oocyst production. Veterinary Parasitolology 149: 147-157.

Salem, S., Azab, M., Soffar, S., Bishara, S. \& El-Kadery, A. (1989). Ultrastructure of penetrating Giardia trophozoite and the possible mechanism of invasion. Journal of the Egyptian Society of Parasitology 19: 461-464.

Samra, N.K., Ganguly, U.C., Garg, J.G. \& Mahajan, R.C. (1988). Effect of excretorysecretory products of Giardia lamblia on glucose and phenylalanine transport in the small intestine of Swiss albino mice. Biochemistry International 17: 801-812.

Shalaby, N.M. \& Wakid, M.H. (2014). Giardiasis in Man: Review and Updates. Journal of King Abdulaziz UniversityMedical Sciences 21: 87-100.

Torgerson, P.R., Devleesschauwer, B., Praet, N., Speybroeck, N., Willingham, A.L., Kasuga, F., Rokni, M.B., Zhou, X.N., Fèvre, E.M., Sripa, B., Gargouri, N., Fürst, T., Budke, C.M., Carabin, H., Kirk, M.D., Angulo, F.J., Havelaar, A. \& de Silva, N. (2015). World Health Organization estimates of the global and regional disease burden of 11 foodborne parasitic diseases, 2010: A data synthesis. PLoS Medicine 12: e1001920.
Toulah, F.H., El-Dardeery, M.A., Al-Shathly, M.R. \& Al-Ahl, S.A.S. (2016). Giardiasis in streptozotocin-induced diabetic C57BL/6 mice. Advanced Environmental Biology 10: 220-231.

Ventura, L.L., Oliveira, D.R., Viana, J.C., Santos, J.F., Caliari, M.V. \& Gomes, M.A. (2013). Impact of protein malnutrition on histological parameters of experimentally infected animals with Giardia lamblia. Experimental Parasitology 133: 391-395.

Wakid, M.H., Azhar, E.I. \& Zafar, T.A. (2009). Intestinal parasitic infection among food handlers in Holy City Makkah during Hajj season 1428 hegira (2007). Journal of King Abdulaziz University-Medical Sciences 16: 39-52.

WHO (World Health Organization), (2020). Available online from: http://www.euro. who.int/_data/assets/pdf_file/0019/ $322165 /$ Situation-water-relatedinfectious-diseases.pdf. [accessed on 10 JAN 2020]. 